\title{
Ion-Acoustic Rogue Waves in Double Pair Plasma Having Non-Extensive Particles
}

\author{
Sharmin Jahan ${ }^{1, *}$, Mohammad Nurul Haque ${ }^{1}$, Nure Alam Chowdhury ${ }^{2}$, Abdul Mannan ${ }^{1,3}$ \\ and Abdullah Al Mamun ${ }^{1}$
}

1 Department of Physics, Jahangirnagar University, Savar, Dhaka 1342, Bangladesh; mnhaque2460@gmail.com (M.N.H.); abdulmannan@juniv.edu (A.M.); mamun_phys@juniv.edu (A.A.M.) Atomic Energy Centre, Plasma Physics Division, Dhaka 1000, Bangladesh; nurealam1743phy@gmail.com 3 Institut für Mathematik, Martin Luther Universität Halle-Wittenberg, D-06099 Halle, Germany

* Correspondence: jahan88phy@gmail.com

check for updates

Citation: Jahan, S.; Haque, M.N.; Chowdhury, N.A.; Mannan, A.; Mamun, A.A. Ion-Acoustic Rogue Waves in Double Pair Plasma Having Non-Extensive Particles. Universe 2021, 7, 63. https://doi.org/ 10.3390/universe7030063

Academic Editor: Lorenzo Iorio

Received: 2 February 2021

Accepted: 9 March 2021

Published: 10 March 2021

Publisher's Note: MDPI stays neutral with regard to jurisdictional claims in published maps and institutional affiliations.

Copyright: (C) 2021 by the authors Licensee MDPI, Basel, Switzerland. This article is an open access article distributed under the terms and conditions of the Creative Commons Attribution (CC BY) license (https:// creativecommons.org/licenses/by/ $4.0 /)$.

\begin{abstract}
The modulational instability (MI) of ion-acoustic (IA) waves (IAWs) and associated IA rogue waves (IARWs) are studied in double-pair plasma containing inertial positive and negative ions, inertialess non-extensive electrons and iso-thermal positrons. A standard nonlinear Schrödinger equation (NLSE) is derived by employing reductive perturbation method. It can be seen from the numerical analysis that the plasma system supports both modulationally stable (unstable) parametric regime in which the dispersive and nonlinear coefficients of the NLSE have opposite (same) sign. It is also found that the basic features of IAWs (viz., MI criteria of IAWs, amplitude, and width of the IARWs, etc.) are rigorously changed by the plasma parameters such as mass, charge state, and number density of the plasma species. The outcomes of our present investigation should be useful in understanding the propagation of nonlinear electrostatic IAWs and associated IARWs in astrophysical and laboratory plasmas.
\end{abstract}

Keywords: ion-acoustic waves; NLSE; modulational instability; rogue waves

\section{Introduction}

Pair-ion (PI) plasma is characterised as fully ionized gas, having electrons, positive and negative ions, and is believed to exist in astrophysical environments such as Van Allen radiation belt and near the polar cap of fast rotation neutron stars [1], solar atmosphere [2], upper regions of Titan's atmosphere [3-10], cometary comae [11], $\left(\mathrm{H}^{+}, \mathrm{O}_{2}^{-}\right)$and $\left(\mathrm{H}^{+}\right.$, $H^{-}$) plasmas in the D and F-regions of Earth's ionosphere [4-9], and also in laboratory experiments namely, $\left(\mathrm{Ar}^{+}, \mathrm{F}^{-}\right)$plasma [12], $\left(\mathrm{K}^{+}, S F_{6}^{-}\right)$plasma [13,14], neutral beam sources [15], plasma processing reactors [16], $\left(\mathrm{Ar}^{+}, S F_{6}^{-}\right)$plasma [17-20], combustion products [21], plasma etching [21], $\left(\mathrm{Xe}^{+}, \mathrm{F}^{-}\right)$plasma [22], $\left(\mathrm{Ar}^{+}, \mathrm{O}_{2}^{-}\right)$plasma, Fullerene $\left(C_{60}^{+}, C_{60}^{-}\right)$plasma [23-25], RF glow discharges [16], and Tokamak [26], etc. A number of authors studied ion-acoustic (IA) waves (IAWs) and associated nonlinear electrostatic structures, namely, solitons, shocks, rogue waves, and double layers in the presence of positrons in PI plasma [27-29].

The Maxwellian distribution function is one of the most widely used velocity distribution functions for describing the dynamics of iso-thermal particles. Highly energetic particles have been observed in the galaxy clusters [30], Earth's bow-shock [31], upper ionosphere of Mars [32], vicinity of the Moon [33], and magnetospheres of Jupiter and Saturn [34], etc. So, to describe the dynamics of these highly energetic particles, Renyi [35] first recognized the modification of Maxwellian distribution, and finally, Tsallis [36] generalized the non-extensive $q$-distribution. It is noted that the parameter $q$ in the nonextensive $q$-distribution characterizes the degree of non-extensivity of these highly energetic particles [37-40]. Shalini et al. [38] studied IAWs in non-extensive plasma having twotemperature electrons, and observed that the width of the first and second-order IA rogue 
waves (IARWs) associated with IAWs decreases with increasing the value of $q$ but the amplitude of the first and second-order IARWs associated with IAWs is remain constant. Tribeche et al. [39] investigated electrostatic solitary waves in the presence of non-extensive electrons, and found that the the amplitude of the potential increases with non-extensive parameter. Hafez and Talukder [40] examined the propagation of nonlinear electrostatic waves in a three-component non-extensive plasma having inertialess non-extensive electrons and positrons, and inertial ions, and reported that the amplitude of the soliton increases with increasing temperature of non-extensive electron.

The investigation of modulational instability (MI) of electrostatic waves is one of the most important research areas for plasma physicists [41,42]. It may be noted that the MI of electrostatic waves is considered to be the primary reason for the formation of massive and gigantic rogue waves (RWs) [43-48]. Rogue wave, which is the rational solution of the standard nonlinear Schrödinger equation (NLSE), is a short-lived phenomenon that emerges from nowhere and disappears without a trace [43-48]. A number of authors have investigated the MI of IAWs by considering the non-extensive particles [49-53]. Bains et al. [51] studied the MI of IAWs in the presence of non-extensive electrons, and demonstrated that the critical wave number $\left(k_{c}\right)$, at which the instability sets in, increases with the increase in the value of $q(q>0)$. Bouzit et al. [52] investigated the stability conditions of IAWs in the presence of non-extensive non-thermal electrons. Eslami et al. [53] investigated the MI of IAWs in electron-positron-ion plasma having non-extensive electrons and positrons, and observed that the $k_{c}$ decreases with $q$ for $q<0$ while increases with $q$ for $q>0$. To the best knowledge of the authors, no attempt has been made to investigate the MI of the IAWs and associated IARWs in a four-component plasma containing inertial positive and negative ions, and inertialess non-extensive electrons, and iso-thermal positrons. Therefore, it is a rational fascination to examine the influence of non-extensive electrons and iso-thermal positrons on the MI of IAWs and associated IARWs in a four-component double pair plasma (DPP).

The manuscript is organized in the following fashion: The model equations are presented in Section 2. The derivation of the NLSE is shown in Section 3. The stability of IAWs is provided in Section 4. The IARWs are demonstrated in Section 5. Finally, a conclusion is given in Section 6.

\section{Model Equations}

We consider the propagation of IAWs in a collisionless, fully ionized, unmagnetized plasma system consisting of warm negative ions (denoted by $n_{-i}$; charge $q_{-i}=-Z_{-i} e$; mass $m_{-i}$ ), warm positive ions (denoted by $n_{+i}$; charge $q_{+i}=Z_{+i} e$; mass $m_{+i}$ ), nonextensive $q$-distributed electrons (denoted by $n_{e}$; charge $q_{e}=-e$; mass $m_{e}$ ), and iso-thermal positrons (denoted by $n_{p}$; charge $q_{p}=+e$; mass $m_{p}$ ); where $Z_{-i}\left(Z_{+i}\right)$ is the charge state of the negative (positive) ion, and $e$ being the magnitude of the charge of electron. The charge neutrality condition of our present model can be written as $n_{p 0}+Z_{+i} n_{+i 0}=n_{e 0}+Z_{-i} n_{-i 0}$. Now, the normalized governing equations can be written as

$$
\begin{aligned}
& \frac{\partial n_{-i}}{\partial t}+\frac{\partial}{\partial x}\left(n_{-i} u_{-i}\right)=0, \\
& \frac{\partial u_{-i}}{\partial t}+u_{-i} \frac{\partial u_{-i}}{\partial x}+\lambda_{1} n_{-i} \frac{\partial n_{-i}}{\partial x}=\frac{\partial \phi}{\partial x}, \\
& \frac{\partial n_{+i}}{\partial t}+\frac{\partial}{\partial x}\left(n_{+i} u_{+i}\right)=0, \\
& \frac{\partial u_{+i}}{\partial t}+u_{+i} \frac{\partial u_{+i}}{\partial x}+\lambda_{3} n_{+i} \frac{\partial n_{+i}}{\partial x}=-\lambda_{2} \frac{\partial \phi}{\partial x}, \\
& \frac{\partial^{2} \phi}{\partial x^{2}}=\lambda_{4} n_{e}+n_{-i}-\left(1+\lambda_{4}-\lambda_{5}\right) n_{p}-\lambda_{5} n_{+i},
\end{aligned}
$$

where $n_{-i}, n_{+i}, n_{e}$, and $n_{p}$ are normalized by $n_{-i 0}, n_{+i 0}, n_{e 0}$, and $n_{p 0}$, respectively; $u_{-i}\left(u_{+i}\right)$ indicates the negative (positive) ion fluid, and is normalized by the IA wave speed $C_{-i}=$ 
$\left(Z_{-i} k_{B} T_{e} / m_{-i}\right)^{1 / 2}$ (with $k_{B}$ being the Boltzmann constant and $T_{e}$ being the temperature of the electron); $\phi$ denotes the electrostatic wave potential, and is normalized by $k_{B} T_{e} / e$; the time and space variables are, respectively, normalized by $\omega_{-i p}^{-1}=\left(m_{-i} / 4 \pi e^{2} Z_{-i}^{2} n_{-i 0}\right)^{1 / 2}$ and $\lambda_{-i}=\left(k_{B} T_{e} / 4 \pi e^{2} Z_{-i} n_{-i 0}\right)^{1 / 2}$. The pressure term of the negative ion can be represented as $P_{-i}=P_{-i 0}\left(N_{-i} / n_{-i 0}\right)^{\gamma}$ with $P_{-i 0}=n_{-i 0} k_{B} T_{-i}$, where $P_{-i 0}\left(T_{-i}\right)$ being the equilibrium pressure (temperature) of negative ion; and $P_{+i}=P_{+i 0}\left(N_{+i} / n_{+i 0}\right)^{\gamma}$ with $P_{+i 0}=n_{+i 0} k_{B} T_{+i}$, where $P_{+i 0}\left(T_{+i}\right)$ being the equilibrium pressure (temperature) of the positive ion, and $\gamma=(N+2) / N$ (where $N$ represents the degree of freedom and for onedimensional case $N=1$, and then $\gamma=3$ ). Other parameters are $\lambda_{1}=3 T_{-i} / Z_{-i} T_{e}, \lambda_{2}=$ $Z_{+i} m_{-i} / Z_{-i} m_{+i}, \lambda_{3}=3 T_{+i} m_{-i} / Z_{-i} m_{+i} T_{e}, \lambda_{4}=n_{e 0} / Z_{-i} n_{-i 0}$, and $\lambda_{5}=Z_{+i} n_{+i 0} / Z_{-i} n_{-i 0}$ Now, the number densities of non-extensive electron [54] and iso-thermal positron can be represented, respectively, by the following normalized equations

$$
\begin{aligned}
& n_{e}=[1+(q-1) \phi]^{\frac{q+1}{2(q-1)}}, \\
& n_{p}=\exp \left(-\lambda_{6} \phi\right),
\end{aligned}
$$

where $\lambda_{6}=T_{e} / T_{p}$ (with $T_{p}$ being the temperature of the iso-thermal positron and $T_{e}>T_{p}$ ). The parameter $q$, generally known as entropic index which defines the degree of nonextensivity. It can be noted that when $q=1$, the entropy reduces to standard MaxwellBoltzmann distribution. On the other hand, in the limits $q>0(q<0)$, the entropy shows sub-extensivity (super-extensivity). Now, by substituting Equations (6) and (7) into Equation (5) and expanding up to third order in $\phi$, we can write

$$
\frac{\partial^{2} \phi}{\partial x^{2}}+1+\lambda_{5} n_{+i}=\lambda_{5}+n_{-i}+M_{1} \phi+M_{2} \phi^{2}+M_{3} \phi^{3}+\cdots,
$$

where

$$
\begin{aligned}
& M_{1}=\left[\lambda_{4}(q+1)+2 \lambda_{6}\left(1+\lambda_{4}-\lambda_{5}\right)\right] / 2 \\
& M_{2}=\left[\lambda_{4}(q+1)(3-q)-4 \lambda_{6}^{2}\left(1+\lambda_{4}-\lambda_{5}\right)\right] / 8 \\
& M_{3}=\left[\lambda_{4}(q+1)(q-3)(3 q-5)+8 \lambda_{6}^{3}\left(1+\lambda_{4}-\lambda_{5}\right)\right] / 48
\end{aligned}
$$

It may be noted here that the terms containing $M_{1}, M_{2}$, and $M_{3}$ in Equation (8) are due to the contribution of non-extensive electrons and iso-thermal positrons.

\section{Derivation of the NLSE}

To study the MI of the IAWs, first we want to derive the NLSE by employing the reductive perturbation method. In that case, the stretched co-ordinates can be written in the following form $[54,55]$

$$
\begin{aligned}
& \xi=\epsilon\left(x-v_{g} t\right), \\
& \tau=\epsilon^{2} t,
\end{aligned}
$$

where $v_{g}$ is the group speed and $\epsilon$ is a small parameter. After that the dependent variables can be represented as $[54,55]$

$$
\Pi(x, t)=\Pi_{0}+\sum_{m=1}^{\infty} \epsilon^{m} \sum_{l=-\infty}^{\infty} \Pi_{l}^{(m)}(\xi, \tau) \exp [i l(k x-\omega t),
$$

where $\Pi_{i l}^{m}=\left[n_{-i l}^{m}, u_{-i l}^{m}, n_{+i l}^{m}, u_{+i l}^{m}, \phi_{i l}^{m}\right], \Pi_{0}=[1,0,1,0,0]^{T}$, and $k(\omega)$ is real variables representing the carrier wave number (frequency). The derivative operators can be written as $[54,55]$ 


$$
\begin{aligned}
\frac{\partial}{\partial t} & \rightarrow \frac{\partial}{\partial t}-\epsilon v_{g} \frac{\partial}{\partial \xi}+\epsilon^{2} \frac{\partial}{\partial \tau}, \\
\frac{\partial}{\partial x} & \rightarrow \frac{\partial}{\partial x}+\epsilon \frac{\partial}{\partial \xi} .
\end{aligned}
$$

Now, by substituting Equations (9) - (13) into Equations (1)-(4) and (8), and collecting the power terms of $\epsilon$, the first order $(m=1$ with $l=1)$ reduced equations can be written as

$$
\begin{aligned}
& n_{-i 1}^{(1)}=\frac{k^{2}}{S} \phi_{1}^{(1)}, \\
& u_{-i 1}^{(1)}=\frac{k \omega}{S} \phi_{1}^{(1)}, \\
& n_{+i 1}^{(1)}=\frac{\lambda_{2} k^{2}}{A} \phi_{1}^{(1)}, \\
& u_{+i 1}^{(1)}=\frac{\lambda_{2} \omega k}{A} \phi_{1}^{(1)},
\end{aligned}
$$

where $S=\lambda_{1} k^{2}-\omega^{2}$ and $A=\omega^{2}-\lambda_{3} k^{2}$. These equations provide the dispersion relation of IAWs in the following form

$$
\begin{aligned}
& \omega^{2} \equiv \omega_{f}^{2}=\frac{I+\sqrt{I^{2}-4 U J}}{2 U}, \\
& \omega^{2} \equiv \omega_{s}^{2}=\frac{I-\sqrt{I^{2}-4 U J}}{2 U},
\end{aligned}
$$

where $I=\left(\lambda_{1} k^{2}+\lambda_{3} k^{2}+\lambda_{1} M_{1}+\lambda_{3} M_{1}+\lambda_{2} \lambda_{5}+1\right), U=\left(k^{2}+M_{1}\right) / k^{2}$, and $J=$ $k^{2}\left(\lambda_{1} \lambda_{3} k^{2}+\lambda_{1} \lambda_{3} M_{1}+\lambda_{3}+\lambda_{1} \lambda_{2} \lambda_{5}\right)$. However, to obtain the real and positive values of $\omega$, the conditions $I^{2}>4 U J$ must be satisfied. It is noted that $\omega_{f}\left(\omega_{s}\right)$ represents the fast (slow) IA modes. The second-order $(m=2$ with $l=1)$ equations and with the compatibility condition, we can write the group velocity of IAWs in the DPP

$$
v_{g}=\frac{\left(\lambda_{2} \lambda_{3} \lambda_{5} k^{2} S^{2}+\lambda_{2} \lambda_{5} \omega^{2} S^{2}+\lambda_{2} \lambda_{5} A S^{2}+\lambda_{1} A^{2} k^{2}+A^{2} \omega^{2}-2 A^{2} S^{2}-S A^{2}\right)}{2 \omega k\left(A^{2}+\lambda_{2} \lambda_{5} S^{2}\right)},
$$

Now, the co-efficient of $\epsilon$ (when $m=2$ with $l=2$ ) yields the second-order harmonic amplitudes, is found to be proportional to $\left|\phi_{1}^{(1)}\right|^{2}$

$$
\begin{aligned}
& n_{-i 2}^{(2)}=M_{4}\left|\phi_{1}^{(1)}\right|^{2}, \\
& u_{-i 2}^{(2)}=M_{5}\left|\phi_{1}^{(1)}\right|^{2}, \\
& n_{+i 2}^{(2)}=M_{6}\left|\phi_{1}^{(1)}\right|^{2}, \\
& u_{+i 2}^{(2)}=M_{7}\left|\phi_{1}^{(1)}\right|^{2}, \\
& \phi_{2}^{(2)}=M_{8}\left|\phi_{1}^{(1)}\right|^{2},
\end{aligned}
$$

where

$$
\begin{aligned}
& M_{4}=\frac{2 M_{8} k^{2} S^{2}-3 \omega^{2} k^{4}-\lambda_{1} k^{6}}{2 S^{3}}, \quad M_{5}=\frac{\omega M_{4} S^{2}-\omega k^{4}}{k S^{2}} \\
& M_{6}=\frac{2 \lambda_{2} M_{8} A^{2} k^{2}+3 \lambda_{2}^{2} \omega^{2} k^{4}+\lambda_{3} \lambda_{2}^{2} k^{6}}{2 A^{3}}, \quad M_{7}=\frac{\omega M_{6} A^{2}-\omega \lambda_{2}^{2} k^{4}}{k A^{2}}, \\
& M_{8}=\frac{2 M_{2} A^{3} S^{3}-3 \lambda_{5} \lambda_{2}^{2} \omega^{2} S^{3} k^{4}-3 \omega^{2} A^{3} k^{4}-\lambda_{3} \lambda_{5} \lambda_{2}^{2} S^{3} k^{6}-\lambda_{1} A^{3} k^{6}}{2 A^{2} S^{2}\left(S \lambda_{2} \lambda_{5} k^{2}-4 k^{2} A S-M_{1} A S-A k^{2}\right)},
\end{aligned}
$$


Now, we consider the expression for $(m=3$ with $l=0)$ and $(m=2$ with $l=0)$, which leads the zeroth harmonic modes. Thus, we obtain

$$
\begin{aligned}
& n_{-i 0}^{(2)}=M_{9}\left|\phi_{1}^{(1)}\right|^{2}, \\
& u_{-i 0}^{(2)}=M_{10}\left|\phi_{1}^{(1)}\right|^{2}, \\
& n_{+i 0}^{(2)}=M_{11}\left|\phi_{1}^{(1)}\right|^{2}, \\
& u_{+i 0}^{(2)}=M_{12}\left|\phi_{1}^{(1)}\right|^{2}, \\
& \phi_{0}^{(2)}=M_{13}\left|\phi_{1}^{(1)}\right|^{2},
\end{aligned}
$$

where

$$
\begin{aligned}
M_{9}= & \frac{2 \omega v_{g} k^{3}+\lambda_{1} k^{4}+k^{2} \omega^{2}-M_{13} S^{2}}{S^{2}\left(v_{g}^{2}-\lambda_{1}\right)}, \quad M_{10}=\frac{M_{9} v_{g} S^{2}-2 \omega k^{3}}{S^{2}}, \\
M_{11}= & \frac{\lambda_{2} M_{13} A^{2}+\lambda_{3} \lambda_{2}^{2} k^{4}+\lambda_{2}^{2} \omega^{2} k^{2}+2 \omega v_{g} \lambda_{2}^{2} k^{3}}{A^{2}\left(v_{g}^{2}-\lambda_{3}\right)}, \\
M_{12}= & \frac{M_{11} v_{g} A^{2}-2 \omega \lambda_{2}^{2} k^{3}}{A^{2}}, \\
M_{13}= & \frac{A^{2}\left(v_{g}^{2}-\lambda_{3}\right)\left(2 \omega v_{g} k^{3}+\lambda_{1} k^{4}+\omega^{2} k^{2}\right)-M_{14}}{A^{2} S^{2} v_{g}^{2}+A^{2} S^{2}\left[\lambda_{2} \lambda_{5}\left(v_{g}^{2}-\lambda_{1}\right)-\lambda_{3}-M_{1}\left(v_{g}^{2}-\lambda_{1}\right)\left(v_{g}^{2}-\lambda_{3}\right)\right]}, \\
M_{14}= & \left(v_{g}^{2}-\lambda_{1}\right) S^{2}\left(2 \lambda_{5} \omega v_{g} \lambda_{2}^{2} k^{3}+\lambda_{3} \lambda_{5} \lambda_{2}^{2} k^{4}+\lambda_{5} \lambda_{2}^{2} \omega^{2} k^{2}\right) \\
& \quad-2 M_{2} A^{2} S^{2}\left(v_{g}^{2}-\lambda_{1}\right)\left(v_{g}^{2}-\lambda_{3}\right) .
\end{aligned}
$$

Finally, the third harmonic modes $(m=3)$ and $(l=1)$, with the help of Equations (14)-(30), give a set of equations, which can be reduced to the following NLSE:

$$
i \frac{\partial \Phi}{\partial \tau}+P \frac{\partial^{2} \Phi}{\partial \xi^{2}}+Q|\Phi|^{2} \Phi=0
$$

where $\Phi=\phi_{1}^{(1)}$ for simplicity. In Equation (31), $P$ is the dispersion co-efficient, which can be written as

$$
\begin{gathered}
P=\frac{4 \lambda_{2} \lambda_{5} \omega^{2} k^{2} v_{g}^{2} S^{3}+4 \lambda_{1} \omega v_{g} A^{3} k^{3}+4 k v_{g} \omega^{3} A^{3}+M_{15}}{2 \omega A S k^{2}\left(A^{2}+\lambda_{2} \lambda_{5} S^{2}\right)}, \\
M_{15}=2 \lambda_{2} \lambda_{3} \lambda_{5} \omega^{2} k^{2} S^{3}+\lambda_{2} \lambda_{5} \lambda_{3}^{2} S^{3} k^{4}+\lambda_{1} S k^{2} A^{3}+\lambda_{2} \lambda_{5} S^{3} \omega^{4}+\lambda_{2} \lambda_{3} \lambda_{5} A k^{2} S^{3} \\
-4 \lambda_{2} \lambda_{5} k v_{g} \omega^{3} S^{3}-4 \lambda_{2} \lambda_{3} \lambda_{5} \omega v_{g} k^{3} S^{3}-4 \omega^{2} k^{2} v_{g}^{2} A^{3}-\lambda_{1}^{2} A^{3} k^{4} \\
-2 \lambda_{1} \omega^{2} k^{2} A^{3}-S k^{2} v_{g}^{2} A^{3}-A^{3} \omega^{4}-\lambda_{2} \lambda_{5} A k^{2} v_{g}^{2} S^{3}-A^{3} S^{3},
\end{gathered}
$$

and $Q$ is the nonlinear co-efficient, which can be written as

$$
Q=\frac{3 M_{3} A^{2} S^{2}+2 M_{2} M_{8} A^{2} S^{2}+2 M_{2} M_{15} A^{2} S^{2}-M_{16}}{2 \omega k^{2}\left(A^{2}+\lambda_{2} \lambda_{5} S^{2}\right)},
$$

where

$$
\begin{aligned}
M_{16} & =2 \omega M_{5} A^{2} k^{3}+2 \lambda_{2} \lambda_{5} \omega M_{7} S^{2} k^{3}+2 \omega M_{10} A^{2} k^{3} \\
& +2 \lambda_{2} \lambda_{5} \omega M_{12} S^{2} k^{3}+\lambda_{1} M_{4} A^{2} k^{4}+M_{4} \omega^{2} A^{2} k^{2} \\
& +\lambda_{2} \lambda_{3} \lambda_{5} M_{6} S^{2} k^{4}+\lambda_{2} \lambda_{5} M_{6} \omega^{2} k^{2} S^{2}+M_{9} \omega^{2} A^{2} k^{2} \\
& +\lambda_{1} M_{9} A^{2} k^{4}+\lambda_{2} \lambda_{5} M_{11} \omega^{2} k^{2} S^{2}+\lambda_{2} \lambda_{3} \lambda_{5} M_{11} S^{2} k^{4}
\end{aligned}
$$


It may be noted here that both $P$ and $Q$ are directly depend on different parameters namely, $\lambda_{1}, \lambda_{2}, \lambda_{3}, \lambda_{4}, \lambda_{5}, \lambda_{6}, q, k$, and are indirectly depend on the mass, number density, temperature, and charge state of the different plasma species.

\section{Modulational Instability of the IAWs}

The propagation of IAWs is modulationaly stable when $P$ and $Q$ have the opposite sign (i.e., $P / Q<0$ ), and is modulationally unstable when both $P$ and $Q$ have same sign (i.e., $P / Q>0)[54,55]$. The point, at which the transition of $P / Q$ curve intersects with the $k$-axis in the " $P / Q$ versus $k^{\prime \prime}$ graph, is known as threshold or critical wave number $k\left(=k_{c}\right)[54,55]$. We have depicted the variation of $P / Q$ with $k$ for different values of $\lambda_{5}$ under the consideration of fast $\left(\omega_{f}\right)$ and slow $\left(\omega_{s}\right)$ IA modes, respectively, in Figures 1 and 2, and it can be seen from these two figures that (a) both stable and unstable parametric regimes are allowed by the plasma system; (b) the $k_{c}$ increases (decreases) with increasing of positive (negative) ion number density when their charge state remains constant; (c) the stable parametric regime (i.e., $P / Q<0$ ) increases (decreases) with positive (negative) ion charge state when their mass remains constant.

The effects of sub-extensivity and super-extensivity of electrons on the stability condition of IAWs can be seen in Figures 3 and 4, respectively. It is obvious from these two figures that the sub-extensive property (i.e., $q>0$ ) of the electrons allows the IAWs to be stable for large wave number while the super-extensive property (i.e., $q<0$ ) of the electrons allows the IAWs to be stable for small a wavenumber, and this result agrees with the result of Eslami et al. [53].

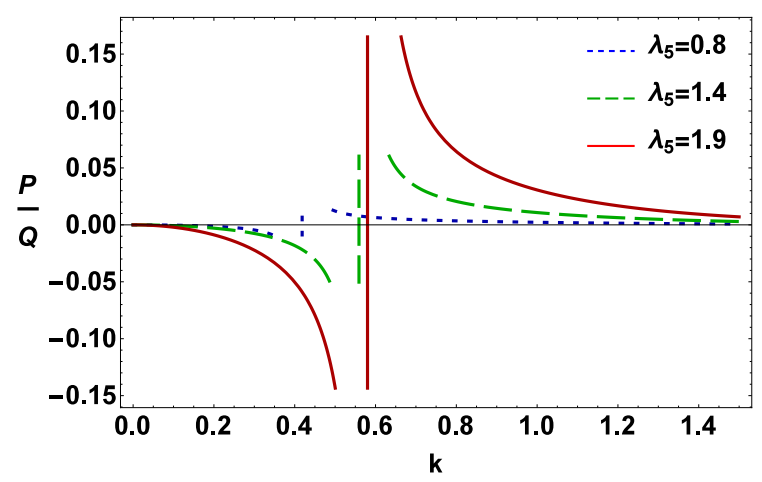

Figure 1. The variation of $P / Q$ with $k$ for different values of $\lambda_{5}$ when $\lambda_{1}=0.007, \lambda_{2}=1.2, \lambda_{3}=0.07$, $\lambda_{4}=1.8, \lambda_{6}=1.7, q=1.4$, and $\omega \equiv \omega_{f}$.

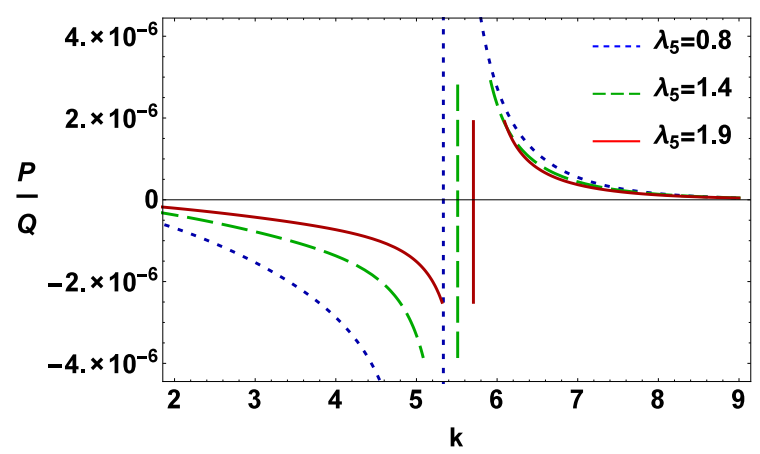

Figure 2. The variation of $P / Q$ with $k$ for different values of $\lambda_{5}$ when $\lambda_{1}=0.007, \lambda_{2}=1.2, \lambda_{3}=0.07$, $\lambda_{4}=1.8, \lambda_{6}=1.7, q=1.4$, and $\omega \equiv \omega_{s}$. 


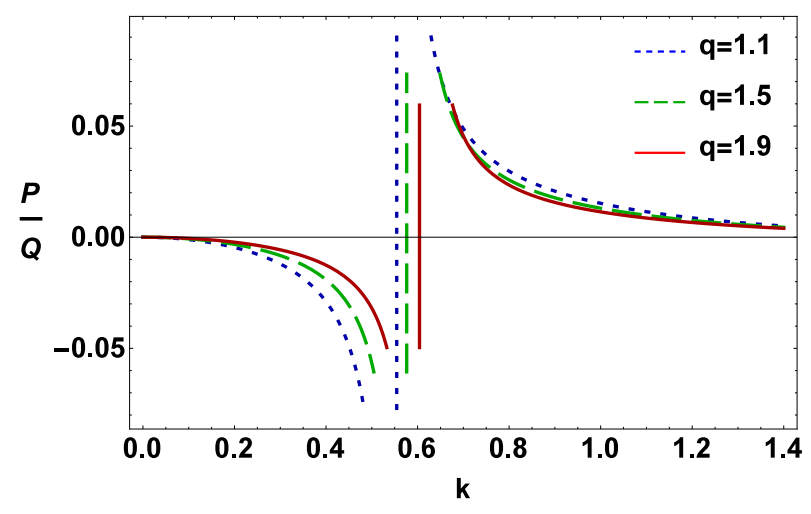

Figure 3. The variation of $P / Q$ with $k$ for different values of $q$ when $\lambda_{1}=0.007, \lambda_{2}=1.2, \lambda_{3}=0.07$, $\lambda_{4}=1.8, \lambda_{5}=1.5, \lambda_{6}=1.7$, and $\omega \equiv \omega_{f}$.

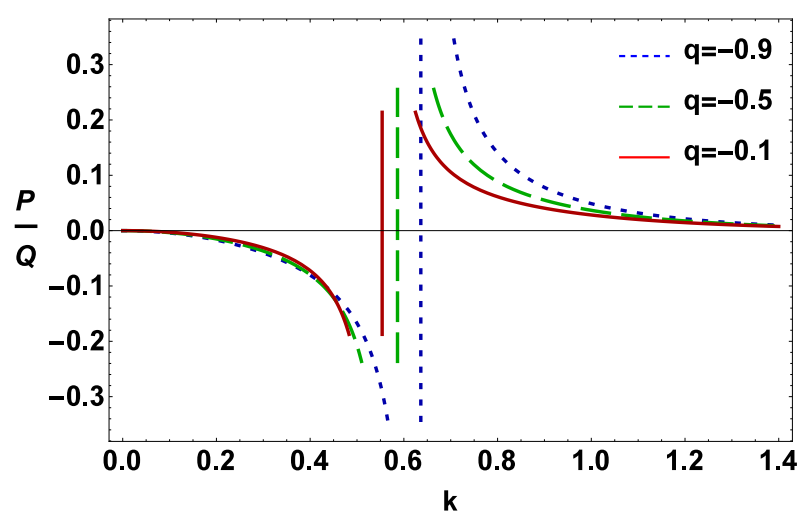

Figure 4. The variation of $P / Q$ with $k$ for different values of $q$ when $\lambda_{1}=0.007, \lambda_{2}=1.2, \lambda_{3}=0.07$, $\lambda_{4}=1.8, \lambda_{5}=1.5, \lambda_{6}=1.7$, and $\omega \equiv \omega_{f}$.

\section{Rogue Waves}

The instability induced from a small perturbation on top of a plane wave leads to an increase in the perturbation up to its highest amplitude and then to a decay so that it finally "disappears without a trace" [43-48]. The NLSE has a variety of solutions, from which the rogue wave/rational solution (developed by Darboux Transformation Scheme) is localized in both the $\xi$ and $\tau$ [43-48]. The first-order rational rogue wave solution of the NLSE in the unstable parametric regime (i.e., $P / Q>0$ ) of the IAWs can be written as [43-48]

$$
\Phi(\xi, \tau)=\sqrt{\frac{2 P}{Q}}\left[\frac{4+16 i \tau P}{1+4 \xi^{2}+16 \tau^{2} P^{2}}-1\right] \exp (2 i \tau P) .
$$

It can be seen from the literature that the PI plasma system can support these conditions: $m_{-i}>m_{+i}$ (i.e., $\mathrm{H}^{+}-\mathrm{O}_{2}^{-}$[4-9], $A r^{+}-S F_{6}^{-}$[17-20], and $\mathrm{X} e^{+}-S F_{6}^{-}$[17-20]), $m_{-i}=$ $m_{+i}$ (i.e., $H^{+}-H^{-}[4-9]$ and $C_{60}^{+}-C_{60}^{-}$[23-25]), and $m_{-i}<m_{+i}$ (i.e., $\left.A r^{+}-F^{-}[5,6]\right)$. So, in our present investigation, we have graphically observed the variation of electrostatic potential with $\lambda_{2}$ under the consideration of $m_{-i}>m_{+i}$ (i.e., $\lambda_{2}>1$ ) in Figure 5 , and it is obvious from this figure that (a) the amplitude and width of the IARWs decrease with an increase in the value of the negative ion mass but increase with an increase in the value of the positive ion mass for a fixed value of their charge state; (b) the height of the IARWs increases (decreases) with negative (positive) ion charge state for a constant mass of positive and negative ion species. So, the mass and charge state of the PI play an opposite role to the formation of IARWs in PI plasama. Figure 6 describes the nature of the electrostatic IARWs with the variation of $\lambda_{2}$ under the consideration of $m_{-i}<m_{+i}$ (i.e., $\lambda_{2}<1$ ). It is clear from this figure that (a) the amplitude and width of IARWs increases (decreases) with negative (positive) ion mass when other plasma parameters are constant; and (b) the existence of 
the heavy positive ion change the dynamics of the plasma system. So, the dynamics of the DPP rigourously changes with these conditions $m_{-i}>m_{+i}$ (i.e., $\lambda_{2}>1$ ) and $m_{-i}<m_{+i}$ (i.e., $\lambda_{2}<1$ ).

The nature of IARWs may also be affected by the electron and positron temperature which can be observed in Figure 7. This figure reveals that as we increase the value of electron (positron) temperature, then the IARWs associated with IAWs are taller (smaller). The physics behind this result is that the nonlinearity of the plasma medium as well as the amplitude of the IARWs decreases (increases) with positron (electron) temperature.

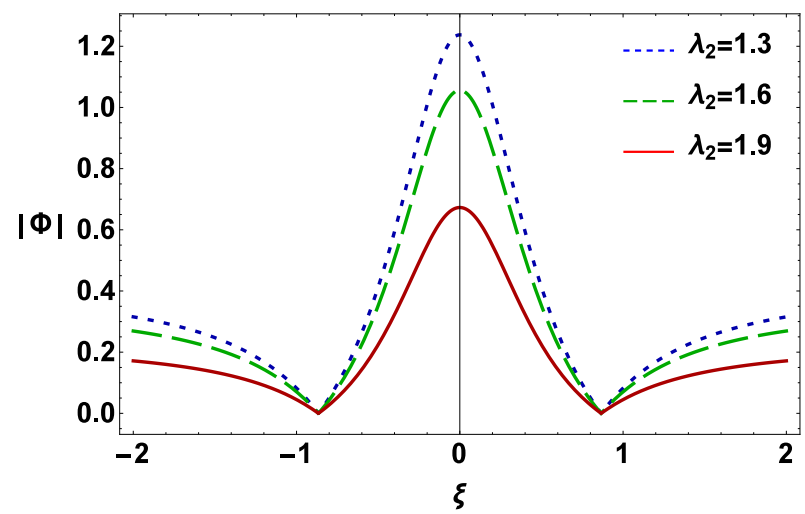

Figure 5. The variation of $|\Phi|$ with $\xi$ for different values of $\lambda_{2}$ when $\lambda_{1}=0.007, \lambda_{3}=0.07, \lambda_{4}=1.8$, $\lambda_{5}=1.5, \lambda_{6}=1.7, \tau=0, k=0.7$, and $\omega \equiv \omega_{f}$.

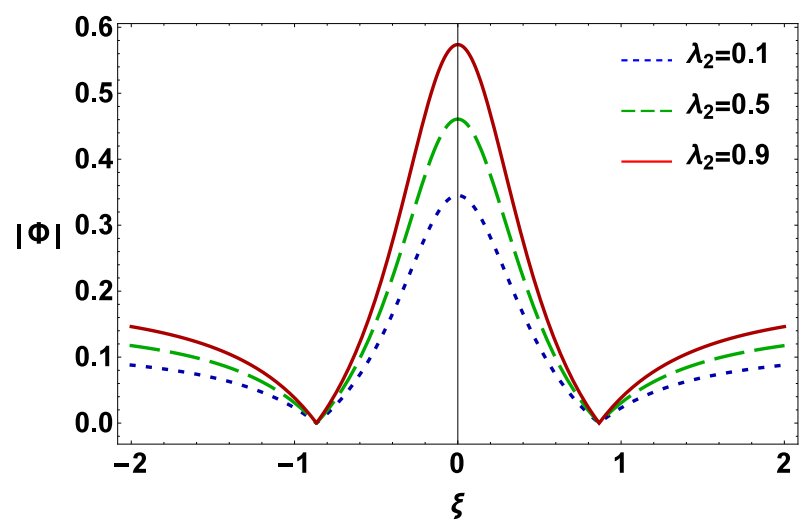

Figure 6. The variation of $|\Phi|$ with $\xi$ for different values of $\lambda_{2}$ when $\lambda_{1}=0.007, \lambda_{3}=0.07, \lambda_{4}=1.8$, $\lambda_{5}=1.5, \lambda_{6}=1.7, \tau=0, k=0.7$, and $\omega \equiv \omega_{f}$.

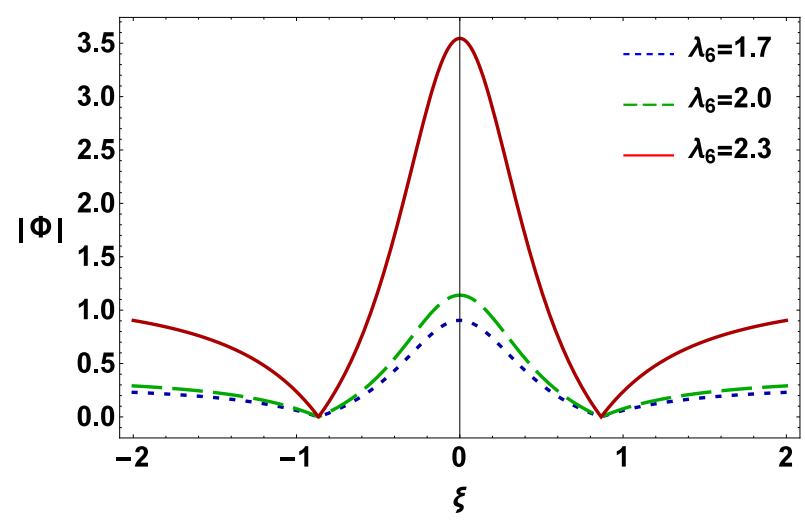

Figure 7. The variation of $|\Phi|$ with $\xi$ for different values of $\lambda_{6}$ when $\lambda_{1}=0.007, \lambda_{2}=1.2, \lambda_{3}=0.07$, $\lambda_{4}=1.8, \lambda_{5}=1.5, \tau=0, k=0.7$, and $\omega \equiv \omega_{f}$. 


\section{Conclusions}

We have investigated the MI of IAWs and associated IARWs in a four-component DPP having inertial positive and negative ions, and inertialess non-extensive electrons and iso-thermal positrons by deriving a standard NLSE. The results that have been found from our investigation can be summarized as follows:

- $\quad$ Both stable and unstable parametric regimes of IAWs can be observed.

- The sub-extensive property of the electrons allows the IAWs to be stable for large wave number while the super-extensive property of the electrons allows the IAWs to be stable for small wave number.

- The dynamics of the DPP rigourously changes with these conditions $m_{-i}>m_{+i}$ (i.e., $\lambda_{2}>1$ ) and $m_{-i}<m_{+i}$ (i.e., $\lambda_{2}<1$ ).

- The nonlinearity of the plasma medium as well as the amplitude of the IARWs decreases (increases) with positron (electron) temperature.

The results of our present investigation are applicable in understanding the process of MI of IAWs and associated IARWs in both astrophysical environments, viz., Van Allen radiation belt and near the polar cap of fast rotation neutron stars [1], solar atmosphere [2], upper regions of Titan's atmosphere [3-10], cometary comae [11], $\left(\mathrm{H}^{+}, \mathrm{O}_{2}^{-}\right)$and $\left(\mathrm{H}^{+}, \mathrm{H}^{-}\right)$plasmas in the D and F-regions of Earth's ionosphere [4-9], and also in laboratory experiments namely, $\left(A r^{+}, F^{-}\right)$plasma [12], $\left(K^{+}, S F_{6}^{-}\right)$plasma [13,14], neutral beam sources [15], plasma processing reactors [16], $\left(A r^{+}, S F_{6}^{-}\right)$plasma [17-20], combustion products [21], plasma etching [21], $\left(\mathrm{Xe}^{+}, \mathrm{F}^{-}\right)$plasma [22], $\left(\mathrm{Ar}+, \mathrm{O}_{2}^{-}\right)$plasma, Fullerene $\left(\mathrm{C}_{60}^{+}, \mathrm{C}_{60}^{-}\right)$plasma [23-25], RF glow discharges [16], and Tokamak [26], etc.

Author Contributions: All authors contributed equally to complete this work. All authors have read and agreed to the published version of the manuscript.

Funding: This research received no external funding.

Institutional Review Board Statement: Not applicable.

Informed Consent Statement: Not applicable.

Data Availability Statement: Data sharing not applicable—no new data generated.

Acknowledgments: The authors are also grateful to the anonymous reviewers for their constructive suggestions which have significantly improved the quality of the manuscript.

Conflicts of Interest: The authors declare no conflict of interest.

\section{References}

1. Lightman, A.P. Relativistic thermal plasmas-Pair processes and equilibria. Astrophys. J. 1982, 253, 842. [CrossRef]

2. Tandberg-Hansen, E.; Emsile, A.G. The Physics of Solar Flares; Cambridge University Press: Cambridge, UK, 1988.

3. Coates, A.J.; Wellbrock, A.; Lewis, G.R.; Jones, G.H.; Young, D.T.; Crary, F.J.; Waite, J.H., Jr. Heavy negative ions in Titan's ionosphere: Altitude and latitude dependence. Geophys. Res. Lett. 2007, 34, L22103.

4. Massey, H. Negative Ions, 3rd ed.; Cambridge University Press: Cambridge, UK, 1976.

5. Sabry, R.; Moslem, W.M.; Shukla, P.K. Fully nonlinear ion-acoustic solitary waves in a plasma with positive-negative ions and nonthermal electrons. Phys. Plasmas 2009, 16, 032302. [CrossRef]

6. Abdelwahed, H.G.; El-Shewy, E.K.; Zahran, M.A.; Elwakil, S.A. On the rogue wave propagation in ion pair superthermal plasma. Phys. Plasmas 2016, 23, 022102. [CrossRef]

7. Misra, A.P. Dust ion-acoustic shocks in quantum dusty pair-ion plasmas. Phys. Plasmas 2009, 16, 033702.

8. Mushtaq, A.; Khattak, M.N.; Ahmad, Z.; Qamar, A. Dust ion acoustic soliton in pair-ion plasmas with non-isothermal electrons. Phys. Plasmas 2012, 19, 042304.

9. Jannat, N.; Ferdousi, M.; Mamun, A.A. Ion-acoustic shock waves in nonextensive multi-Ion plasmas. Commun. Theor. Phys. 2015, 64, 479. [CrossRef]

10. El-Labany, S.k.; Behery, E.E.; El-Razek, S.N.A.; Abdelrazek, L.A. Shock waves in magnetized electronegative plasma with nonextensive electrons. Eur. Phys. J. D 2020, 74, 104. [CrossRef]

11. Chaizy, P.H.; Reme, H.; Sauvaud, J.A.; d’Uston, C.; Lin, R.P.; Larson, D.E.; Mitchell, D.L.; Anderson, K.A.; Carlson, C.W.; Korth, A.; et al. Negative ions in the coma of comet Halley. Nature 1991, 349, 393. [CrossRef]

12. Nakamura, Y.; Tsukabayashi, I. Observation of modified Korteweg—de Vries solitons in a multicomponent plasma with negative Ions. Phys. Rev. Lett. 1984, 52, 2356. [CrossRef] 
13. Song, B.; DAngelo, N.; Merlino, R.L. Ion-acoustic waves in a plasma with negative ions. Phys. Fluids B 1991, 3, 284. [CrossRef]

14. Sato, N.; Production of negative ion plasmas in a Q-machine. Plasma Sources Sci. Technol. 1994, 3, 395. [CrossRef]

15. Bacal, M.; Hamilton, G.W. $H^{-}$and $D^{-}$Production in Plasmas. Phys. Rev. Lett. 1979, 42, 1538. [CrossRef]

16. Gottscho, R.A.; Gaebe, C.E. Negative ion kinetics in RF glow discharges. IEEE Trans. Plasma Sci. 1986, 14, 92. [CrossRef]

17. Wong, A.Y.; Mamas, D.L.; Arnush, D. Negative ion plasmas. Phys. Fluids 1975, 18, 1489. [CrossRef]

18. Nakamura, Y.; Odagiri, T.; Tsukabayashi, I. Ion-acoustic waves in a multicomponent plasma with negative ions. Plasma Phys. Control. Fusion 1997, 39, 105. [CrossRef]

19. Cooney, J.L.; Gavin, M.T.; Lonngren, K.E. Experiments on Korteweg-de Vries solitons in a positive ion-negative ion plasma. Phys. Fluids B 1991, 3, 2758. [CrossRef]

20. Nakamura, Y.; Bailung, H.; Lonngren, K.E. Oblique collision of modified Korteweg-de Vries ion-acoustic solitons. Phys. Plasmas 1999, 6, 3466. [CrossRef]

21. Sheehan, D.P.; Rynn, N. Negative-ion plasma sources. Rev. Sci. Instrum. 1988, 59, 8. [CrossRef]

22. Ichiki, R.; Yoshimura, S.; Watanabe, T.; Nakamura, Y.; Kawai, Y. Experimental observation of dominant propagation of the ion-acoustic slow mode in a negative ion plasma and its application. Phys. Plasmas 2002, 9, 4481.

23. Oohara, W.; Hatakeyama, R. Pair-ion Plasma generation using Fullerenes. Phys. Rev. Lett. 2003, 91, 205005. [CrossRef]

24. Hatakeyama, R.; Oohara, W. Properties of Pair-ion plasmas using Fullerenes. Phys. Scripta 2005, 116, 101. [CrossRef]

25. Oohara, W.; Date, D.; Hatakeyama, R. Electrostatic waves in a paired Fullerene-ion plasma. Phys. Rev. Lett. 2005, 95, 175003. [CrossRef] [PubMed]

26. Helander, P.; Ward, D.J. Positron Creation and Annihilation in Tokamak Plasmas with Runaway Electrons. Phys. Rev. Lett. 2003, 90, 135004. [CrossRef] [PubMed]

27. Esfandyari-Kalejahi, A.; Kourakis, I.; Shukla, P.K. Oblique modulation of electrostatic modes and envelope excitations in pair-ion and electron-positron plasmas. Phys. Plasmas 2006, 13, 122310. [CrossRef]

28. Abdelsalam, U.M.; Moslem, W.M.; Shukla, P.K. Ion-acoustic solitary waves in a dense pair-ion plasma containing degenerate electrons and positrons. Phys. Lett. A 2008, 372, 4057. [CrossRef]

29. Sabry, R. Modulation instability of ion thermal waves in a pair-ion plasma containing charged dust impurities. Phys. Plasma 2008, 15, 092101. [CrossRef]

30. Hansen, S.H. Cluster temperatures and non-extensive thermo-statistics. New Astron. 2005, 10, 371. [CrossRef]

31. Asbridge, J.R.; Bame, S.J.; Strong, I.B. Outward flow of protons from the Earth's bow shock. J. Geophys. Res. $1968,73,5777$. [CrossRef]

32. Lundlin, R.; Zakharov, A.; Pellinen, R.; Borg, H.; Hultqvist, D.; Pissarenko, N.; Dubinin, E.M.; Barabash, S.W.; Liede, I.; Koskinen, H. First measurements of the ionospheric plasma escape from Mars. Nature 1989, 341, 609. [CrossRef]

33. Futaana, Y.; Machida, S.; Saito, Y.; Matsuoka, A.; Hayakawa, H. Moon-related nonthermal ions observed by Nozomi: Species, sources, and generation mechanisms. J. Geophys. Res. 2003, 108, 1025. [CrossRef]

34. Krimigis, S.M.; Carbary, J.F.; Keath, E.P.; Armstrong, T.P.; Lanzerotti, L.J.; Gloeckler, G. General characteristics of hot plasma and energetic particles in the Saturnian magnetosphere: Results from the Voyager spacecraft. J. Geophys. Res. 1983, 88, 8871.

35. Renyi, A. On a new axiomatic theory of probability. Acta Math. Acad. Sci. Hung. 1955, 6, 285. [CrossRef]

36. Tsallis, C. Possible generalization of Boltzmann-Gibbs statistics. J. Stat. Phys. 1988, 52, 479. [CrossRef]

37. Wang, Y.Y.; Li, J.T.; Dai, C.Q.; Chen, V.F. Solitary Waves Rogue Waves A Plasma Nonthermal Electrons Featur. Tsallis Distribution. Phys. Lett. A 2013, 377, 2097. [CrossRef]

38. Shalini; Saini, N.S.; Misra, A.P. Modulation of ion-acoustic waves in a nonextensive plasma with two-temperature electrons. Phys. Plasmas 2015, 22, 092124. [CrossRef]

39. Tribeche, M.; Djebarni, L.; Amour, R. Ion-acoustic solitary waves in a plasma with a q-nonextensive electron velocity distribution. Phys. Plasmas 2010, 17, 042114. [CrossRef]

40. Hafez, M.G.; Talukder, M.R. Ion acoustic solitary waves in plasmas with nonextensive electrons, Boltzmann positrons and relativistic thermal ions. Astrophys. Space Sci. 2015, 359, 27. [CrossRef]

41. Lesur, M.; Diamond, P.H.; Kosuga, Y. Nonlinear current-driven ion-acoustic instability driven by phase-space structures. Plasma Phys. Control. Fusion 2014, 56, 075005.

42. Berman, R.H.; Tetreault, D.J.; Dupree, T.H. Simulation of phase space hole growth and the development of intermittent plasma turbulence. Phys. Fluids 1985, 28, 155. [CrossRef]

43. Akhmediev, N.; Ankiewiez, A.; Soto-Crespo, J.M. Rogue waves and rational solutions of the nonlinear Schrödinger equation. Phys. Rev. E 2009, 80, 026601. [CrossRef] [PubMed]

44. Anikiewicz, A.; Devine, N.; Akhmediev, N. Are rogue waves robust against perturbations? Phys. Lett. A 2009, $373,3997$. [CrossRef]

45. Akhmediev, N.; Soto-Crespo, J.M.; Ankiewicz, A. Extreme waves that appear from nowhere: On the nature of rogue waves. Phys. Lett. A 2009, 373, 2137. [CrossRef]

46. Akhmediev, N.; Soto-Crespo, J.M.; Ankiewicz, A. How to excite a rogue wave. Phys. Rev. A 2009, 80, 043818. [CrossRef]

47. Guo, S.; Mei, L. Modulation instability and dissipative rogue waves in ion-beam plasma: Roles of ionization, recombination, and electron attachment. Phys. Plasmas 2014, 21, 112303. [CrossRef]

48. Kedziora, D.; Ankiewiez A.; Akhmediev, N. Circular rogue wave clusters. Phys. Rev. E 2011, 84, 056611. [CrossRef] [PubMed] 
49. Haque, M.N.; Mannan, A.; Mamun, A.A. Three-dimensional Nonlinear Structures in Magnetized Complex Plasmas. Plasma Phys. Rep. 2019, 45, 1026. [CrossRef]

50. Haque, M.N.; Mannan, A.; Mamun, A.A. The (3+1) dimensional dust-acoustic waves in multi-components magneto-plasmas. Contrib. Plasma Phys. 2019, 59, e201900049. [CrossRef]

51. Bains, A.S.; Tribeche, M.; Gill, T.S. Modulational instability of ion-acoustic waves in a plasma with a q-nonextensive electron velocity distribution. Phys. Plasmas 2011, 18, 022108. [CrossRef]

52. Bouzit, O.; Tribeche, M.; Bains, A.S. Modulational instability of ion-acoustic waves in plasma with a q-nonextensive nonthermal electron velocity distribution. Phys. Plasmas 2015, 22, 084506. [CrossRef]

53. Eslami, P.; Mottaghizadeh, M.; Pakzad, H.R. Modulational instability of ion acoustic waves in e-p-i plasmas with electrons and positrons following a q-nonextensive distribution. Phys. Plasmas 2011, 18, 102313. [CrossRef]

54. Chowdhury, N.A.; Mannan, A.A.; Hasan, M.M.; Mamun, A.A. Heavy ion-acoustic rogue waves in electron-positron multi-ion plasmas. Chaos 2017, 27, 093105.

55. Banik, S.; Shikha, R.K.; Noman, A.A.; Chowdhury, N.A.; Mannan, A.; Roy, T.S.; Mamun, A.A. First and second-order dust-ionacoustic rogue waves in non-thermal plasma. Eur. Phys. J. D 2021, 75, 43. [CrossRef] 